\title{
Reflexive Visual Orienting in Response to the Social Attention of Others
}

\author{
Stephen R. H. Langton and Vicki Bruce
}

Department of Psychology, University of Stirling

Running Head: VISUAL ORIENTING AND SOCIAL ATTENTION

Correspondence to: Dr. S. R. H. Langton, Dept. of Psychology, University of Stirling, Stirling, FK9 4LA, Scotland.

E-Mail: srhl1@stirling.ac.uk

Fax: +44 (0) 1786-467-641 


\begin{abstract}
Four experiments investigate the hypothesis that cues to the direction of another's social attention produce a reflexive orienting of an observer's visual attention. Participants were asked to make a simple detection response to a target letter which could appear at one of four locations on a visual display. Prior to the presentation of the target, one of these possible locations was cued by the orientation of a digitised head stimulus which appeared at fixation in the centre of the display. Uninformative and to-be-ignored cueing stimuli produced faster target detection latencies at cued relative to uncued locations, but only when the cues appeared $100 \mathrm{~ms}$ before the onset of the target (Experiments 1 and 2). The effect was uninfluenced by the introduction of a to-be-attended and relatively informative cue (Experiment 3), but was disrupted by the inversion of the head cues (Experiment 4). It is argued that these findings are consistent with the operation of a reflexive, stimulus-driven or exogenous orienting mechanism which can be engaged by social attention signals.
\end{abstract}


Consider a social interaction between a small group of people. The members of this group will constantly enter and exit the conversational floor, rapidly exchanging the roles of speaker and listener as the conversation progresses. During the interaction a speaker might direct his or her remarks to a particular individual whilst simultaneously referring to other members of the group, to other persons, or to objects of interest in the environment. In order to follow the ebb and flow of this type of social interaction, to understand who is talking to whom, or to grasp the referent of some remark, two main processes seem to be necessary. One must first make sense of the various social signals which a speaker or a listener might use to indicate the focus of their social attention, and second, shift the focus of one's own attention rapidly and efficiently in accord with these signals. The experiments reported in this paper focus on this latter orienting process. These studies explore the possibility that social attention signals trigger a reflexive shift of an observer's visual attention.

\section{Social Attention Perception}

The direction in which another individual is directing their attention is of particular importance to many species. For instance, humans use gaze to signal turns in conversation and to communicate interpersonal attitudes such as love or dominance (see Kleinke, 1986 for a review). Similarly animals use gaze as signals of threat, appeasement or affiliation. In addition, a significant environmental event such as the approach of a predator, a potential mate or a meal will often be signalled by a sudden change in another's gaze, head orientation or body posture. Thus, it would appear to be of particular adaptive advantage for animals to monitor the attentional signals provided by other individuals.

Neuropsychological and neurophysiological evidence is beginning to indicate that there are functionally separate mechanisms devoted to the task of perceiving where another's attention is directed. For instance, dissociations have been noted in brain injured patients between face recognition and gaze perception abilities (e.g., Campbell, Heywood, Cowey, Regard \& Landis, 1990; Perrett, Mistlin, Chitty, Harries, Newcombe \& de Haan, 1988) suggesting that these two 
aspects of face processing proceed independently in the information processing system. Other work suggests that gaze detection is one of the functions of the superior temporal sulcus (STS) region of the primate temporal lobe. Heywood and Cowey (1992) showed that the removal of this region of the macaque cortex impaired gaze direction judgements by these monkeys. These findings seem to indicate the existence of a functionally separate gaze detection module. Indeed, Baron-Cohen (1994, 1995a, 1995b) has proposed just such a "neurocognitive mechanism" as part of his "mindreading" system; a collection of modules which have evolved to enable humans to attribute mental states to other agents. His "Eye Direction Detector" (EDD) functions to detect eyes, or eye-like stimuli, and also computes whether eyes are directed toward it or toward another object in the environment.

However, Perrett and his colleagues have suggested that something like an EDD comprises only part of a system designed to process the direction of "social attention" (e.g., Perrett \& Emery, 1994; Perrett, Hietanen, Oram \& Benson, 1992). Their work has indicated that individual cells in the STS region of the macaque brain respond to conjunctions of eye, head and body position. Thus, cells which respond preferentially to eyes directed downwards show further preferences when the head is oriented downwards and when the body adopts a quadrupedal posture. Accordingly, Perrett et al. (1992) suggest that one function of the STS region lies in the analysis of social attention direction. They propose a more general "Direction of Attention Detector" (DAD) which combines information from separate detectors analysing body, head and gaze direction (Perrett \& Emery, 1994). Two recent studies have provided some evidence in support of the proposal that information from the head can influence the processing of gaze direction. Vecera and Johnson (1995) showed that participants' sensitivity to gaze direction in schematic faces was poorer when the eyes were in the context of an inverted or scrambled face compared to an upright face. More recently, Jenkins (in prep) obtained a similar result using photographs of real faces, but also demonstrated that sensitivity to gaze direction was compromised even when the eyes remained upright in the context of an inverted face. 
A number of authors have suggested, largely on the basis of anecdote or introspection, that having established where another individual is directing their attention, we have some kind of compulsive tendency to then align our own attention to the same spatial location (e.g., BaronCohen, 1994; Langton, 1996; Tantam, 1992). This kind of response would seem to be of obvious adaptive advantage as the deployment of attention to locations of interest has been shown to enhance the processing of stimuli occupying these positions (see below). Thus, by following another's gaze, the detection or discrimination of an intruder will be facilitated allowing more time for the programming and execution of an appropriate course of action. Accordingly, Tantam (1992) argues that there is "an innate and involuntary orientation to social stimuli" (p.84). That is, a compulsive shift in gaze toward faces, facial expressions and human voices. Futhermore, Tantam proposes that a "second gaze response" develops which ensures that a child's gaze is drawn from, say, the mother's eyes to the object at which she is looking. Baron-Cohen (1994) goes further, arguing that this kind of joint or shared attention is established by the operation of a specialised neurocognitive mechanism which forms an additional component to his mindreading system. This so-called Shared Attention Mechanism (SAM) is concerned with representing the situation when both the self and another are attending to the same object or event. Insofar as the SAM and the other neurocognitive mechanisms comprising the mindreading system operate much like traditional Fodorian modules (Fodor, 1983), it follows that the detection of another's attention signals, and the subsequent visual orienting in response to these cues, should be rapid and obligatory in nature (Baron-Cohen, 1994).

However, the evidence for a compulsive orienting to attentional signals is sparse. At the neuroanatomical level, Harries \& Perrett (1991) noted that the STS region, comprising the putative direction of attention detector, supplies heavy output projections to the parietal cortex which is implicated in attentional orienting (see Posner \& Peterson, 1990; Rafal, 1996 for reviews). Perrett and his colleagues (e.g., Perrett et al., 1992; Walsh and Perrett, 1994) speculate that this link may provide a channel through which another individual's attention can influence the attentional orientation of an observer. A number of developmental studies have indicated that 
infants as young as two to six months tend to adjust their own attention contingent on a change in the focus of attention of the adult (e.g., Butterworth \& Jarrett, 1991; Scaife \& Bruner, 1975; but see Corkum \& Moore, 1995). However, it is unclear whether the incidences of gaze following in these studies are reflexive or automatic in nature. Moreover, joint attention or gaze following is often indexed by an overt head turn or gaze on behalf of the child. This overlooks the possibility that the visual orienting involved in the establishment of joint or shared attention could be the result of a covert orienting process, that is, a shift of attention without any accompanying movement of the eyes or head (see below). Accordingly, in the present studies we adopted a technique widely used in the study of both overt and covert visual orienting. The aim was to examine whether normal adult humans shift their attention reflexively in line with another's gaze or head orientation.

\section{Visual Orienting}

Visual orienting is typically demonstrated and studied using the cueing paradigm introduced by Posner (e.g., 1980). In experiments of this kind, participants are asked to make a simple keypress response to the onset of a visual target which can appear at one of several locations in a display. Before the onset of the target stimulus, the participant's attention is directed to one of the possible target locations by some kind of cue, such as an arrow in the centre of the screen, or a flash of light in the periphery. The typical finding is that target detection (e.g., Posner, 1980; Rafal, Calabresi, Brennan \& Sciolto, 1989) or discrimination (e.g., Cheal \& Lyon, 1991; Erikson \& Collins, 1969; Müller \& Rabbitt, 1989) is faster at the location indicated by the cue, relative to the uncued locations. Not only does this cueing effect occur when participants make an overt orienting response by moving their eyes in the direction of the cue (e.g., Posner, 1980), but also when participants are instructed not to move their eyes, or when the interval between the onset of the cue and the offset of the target is too brief to allow the programming and execution of a saccadic eye-movement (e.g., Posner, 1980; Posner, Snyder \& Davidson, 1980). The advantage for cued over non-cued locations in the absence of eye-movements is usually attributed to a shift in covert visual attention. 
In addition to the overt/covert distinction, researchers have also delineated two qualitatively different mechanisms underlying visual orienting (e.g., Briand \& Klein, 1987; Jonides, 1981; Müller \& Rabbitt, 1989). The first is a fast reflexive or exogenous mechanism which is engaged by the abrupt onset of a visual cue in the periphery of the visual field. This form of orienting is considered to be purely under the control of the cueing stimulus, and can occur even when the cue is completely uninformative, or even counter-informative, regarding the likely location of the target. In contrast, the endogenous mechanism is slower and under strategic or voluntary control. It can be engaged by a predictive event, such as a centrally presented arrow which indicates the probable location of the target. The distinction between these two orienting mechanisms is made on the basis of a number of findings. For instance, exogenous orienting is not disrupted by a secondary memory task that nevertheless affects endogenous orienting to a central cue (Jonides, 1981). Exogenous orienting also appears to be resistant to suppression (Jonides, 1981) and less subject to interruption by subsequent visual events (Müller \& Rabbitt, 1989). Furthermore, the cueing effects produced by the two orienting mechanisms appear to have different time courses. Exogenous orienting produces cueing effects that peak at cue-target stimulus onset asynchronies (SOAs) of around 100 - 150 ms (e.g., Müller \& Findlay, 1988; Müller \& Rabbitt, 1989). The effects produced by endogenous orienting, on the other hand, require 300 - $400 \mathrm{~ms}$ to reach their maximum. Exogenous orienting is further characterised by a biphasic effect so that at SOAs of longer than $300 \mathrm{~ms}$ the detection and discrimination of targets at previously cued locations seems to be inhibited, producing longer RTs on cued than on uncued trials (Lupiáñez, Milán, Tornay, Madrid \& Tudela, 1997; Maylor \& Hockey; 1985; Posner \& Cohen, 1984; Pratt, Kingstone \& Khoe, 1997; Rafal et al., 1989). This so-called inhibition of return (IOR) effect does not normally occur with endogenously activated covert shifts of attention in response to central symbolic cues. In this case normal cueing effects are maintained at SOAs of longer than $400 \mathrm{~ms}$ (e.g., Müller \& Findlay, 1988; Müller \& Rabbitt, 1989).

The experiments reported here use the cueing paradigm to explore the hypothesis that another's visual attention cues produce an exogenous reflexive orienting of an observers visual 
attention. In these experiments, participants were asked to make a speeded keypress response upon the detection of a target letter which could appear either above, below, or to the left or right of fixation. These targets were preceded by a precue which consisted of a head oriented toward one of the possible target locations. The literature on the development of joint visual attention in children has indicated that a combined change in head and eye orientation is a more effective cue for joint attention, that is, for overt head turning in response to these cues (e.g., Butterworth \& Jarrett, 1991; Corkum \& Moore, 1995; Lempers, 1979). Thus, the cueing stimuli in all experiments comprised both head and eye signals. Although these cues are more akin to the symbolic central cues (e.g., arrows or arrowheads) which are thought to engage only the endogenous system, the prediction is that they will also induce some kind of exogenous orienting response. This hypothesis was examined in the four experiments reported below. In Experiment 1 the head cues were $100 \%$ informative of the axis on which the target could appear, but only indicated its exact location on this axis $50 \%$ of the time. This cue validity was reduced to $25 \%$ in Experiment 2 so that the cues were completely uninformative of the likely target location. Experiment 3 examined whether orienting effects obtained in Experiments 1 and 2 could be influenced by participants' knowledge or intentions. This was achieved by asking participants to attend to the head cues which, in this case, were relatively informative regarding the likely location of the target. Finally, in Experiment 4 the cues were inverted in order to examine the hypothesis that the orienting effects observed in Experiments 1-3 were triggered by the direction of attention signalled by the cues, or were the result of some physical property of the cues which is confounded with their direction of attention.

\section{Experiment 1}

In this experiment the head precues correctly predicted the subsequent location of the target on only $50 \%$ of the trials. In this respect the cues were uninformative regarding the exact location of the target stimulus. Therefore, any advantage for target detection at cued locations over uncued locations will presumably represent the operation of a reflexive or exogenous redirection of attention to the location indicated by the head precue. Stimulus onset asynchronies 
(SOAs) of 100, 500 and $1000 \mathrm{~ms}$ were used in this experiment. If the head cues produce the kind of exogenous orienting of attention normally associated with peripheral stimulation, cueing effects might be expected in the $100 \mathrm{~ms}$ SOA condition. However, as the advantage for target detection on cued versus uncued trials typically dissipates by around $300 \mathrm{~ms}$ (see above), no effects were expected in the $500 \mathrm{~ms}$ or $1000 \mathrm{~ms}$ SOA conditions.

\section{Method}

Participants. These were 32 undergraduates recruited through advertisements. All with normal or corrected-to-normal vision.

Apparatus and Materials. Cues and targets were presented using SuperLab software on a Macintosh Performa 630. Participants were seated approximately $0.6 \mathrm{~m}$ from a 14 inch monitor. Cues consisted of digitised images of a head directed up, down, left and right (see Figure 1). These cues subtended a vertical angle of $5^{\circ}$ and $4^{\circ}$ of horizontal visual angle. Targets consisted of a lower case letter "o" in 18 point times, positioned $6^{\circ}$ above, below, to the left or right of a fixation cross located in the centre of the screen.

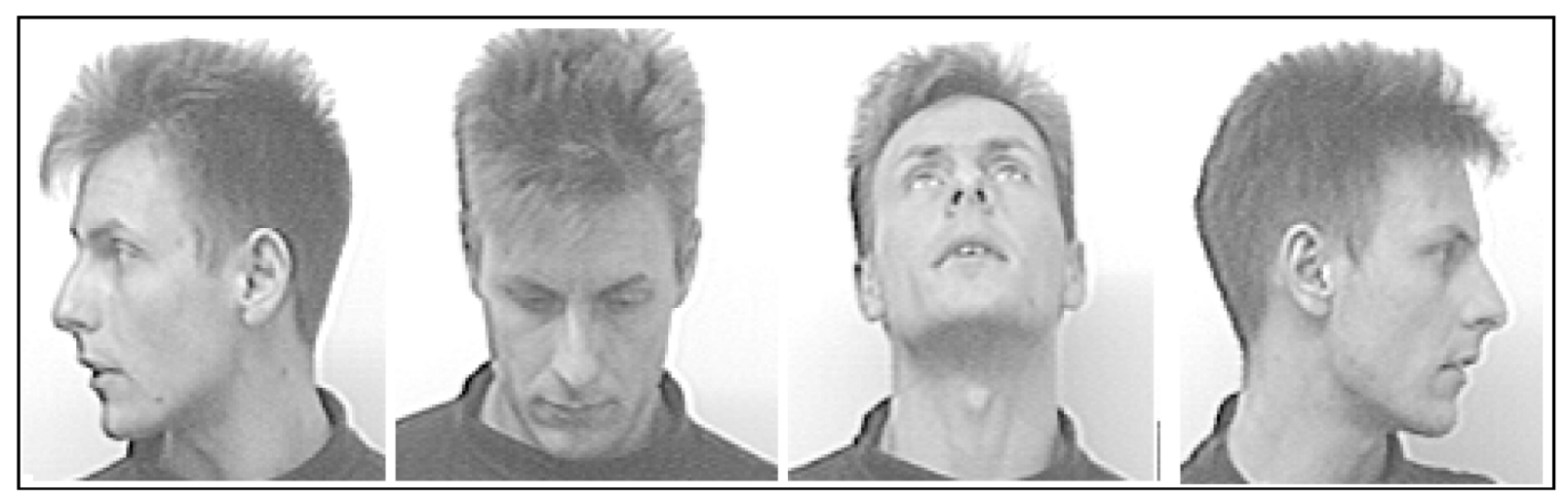

Figure 1. The stimuli used as cues in Experiments 1-4.

Design. There were three within-subjects factors: Cue Condition (valid or invalid), Stimulus Onset Asynchrony (SOA) between cue and target (100 ms, $500 \mathrm{~ms}$ and $1000 \mathrm{~ms}$ ), and Cue Axis (horizontal or vertical cues). These factors were crossed to yield twelve experimental conditions. 
Procedure. At the beginning of each trial a fixation cross appeared in the centre of the screen. Participants were asked to fixate on this cross and to maintain fixation at the centre of the screen throughout the experiment. After a delay of $2000 \mathrm{~ms}$ the fixation cross was replaced by the head cue which also appeared in the centre of the screen. The cue remained on the screen for either $100 \mathrm{~ms}, 500 \mathrm{~ms}$ or $1000 \mathrm{~ms}$ depending on the SOA condition. Following the SOA the cue was replaced by the fixation cross and the target which was presented at one of four locations on the screen. Targets always appeared on the same axis as the cue, that is, following a "left" cue the target would either appear in the left location (valid trial) or the right location (invalid trial). Targets were equally likely to appear in the cued or uncued location along the appropriate axis. Participants were told to ignore the head cues as they did not predict the likely location of the forthcoming target, and were asked to press the space bar on the keyboard with their preferred hand as quickly as possible when they detected the target letter. Following the response, the target disappeared and the screen remained blank for $1000 \mathrm{~ms}$ before the next trial began. Participants completed two blocks of 96 trials. Each block consisted of four repetitions of each of the twelve experimental conditions with all four cues presented once within each condition. Each block also contained a set of 48 "catch" trials where no target was presented. On these trials participants were asked to refrain from making a response. The aim of these trials was to discourage anticipatory responses. Prior to the two experimental blocks, participants completed a sequence of 16 practice trials which included examples of each of the experimental conditions as well as catch trials. Trials were randomised within all blocks.

\section{Results}

Median RTs were calculated for each of the twelve experimental conditions for every participant. The interparticipant means of these median RTs are shown in Table 1, together with the associated standard deviations. There is an overall $13 \mathrm{~ms}$ effect of cue condition at the 100 ms SOA which is reduced to $0 \mathrm{~ms}$ at $500 \mathrm{~ms} \mathrm{SOA}$ and $1 \mathrm{~ms}$ at $1000 \mathrm{~ms}$ SOA. Overall RT's increase as the cue to target interval increases. The average rate of catch trial errors was $1.24 \%$. 
Table 1

Means of Median Reaction Times (RTs; in milliseconds) and Standard Deviations for Detecting Targets Cued by Horizontal and Vertical Valid and Invalid Heads in Experiment 1.

\begin{tabular}{ccccccc}
\hline & \multicolumn{5}{c}{ Stimulus Onset Asynchrony } \\
\cline { 2 - 7 } Cue Axis & \multicolumn{2}{c}{$100 \mathrm{~ms}$} & \multicolumn{2}{c}{$500 \mathrm{~ms}$} & \multicolumn{2}{c}{$1000 \mathrm{~ms}$} \\
\cline { 2 - 7 } & Valid Cue & Invalid Cue & Valid Cue & Invalid Cue & Valid Cue & Invalid Cue \\
\hline Horizontal & & & & & \\
RT & 361 & 376 & 378 & 383 & 391 & 394 \\
SD & 44 & 44 & 42 & 54 & 47 & 56 \\
Vertical & & & & & & \\
RT & 364 & 375 & 388 & 383 & 390 & 390 \\
SD & 48 & 42 & 48 & 48 & 45 & 46 \\
Average & & & & & & \\
RT & 363 & 376 & 383 & 383 & 391 & 392 \\
SD & 46 & 43 & 44 & 51 & 46 & 51 \\
\hline
\end{tabular}

A 2 (Cue Condition) x 3 (SOA) x 2 (Cue Axis) analysis of variance (ANOVA) conducted on the RT data yielded a main effect of $\operatorname{SOA}(\mathrm{F}(2,62)=9.88, \mathrm{p}<0.001)$ and a significant SOA $\mathrm{x}$ Cue Condition interaction $(\mathrm{F}(2,62)=3.38, \mathrm{p}<0.05)$. No other effects or interactions reached significance (p's > 0.1). Simple main effects analysis of the SOA x Cue Condition interaction confirmed that the effect of cue condition was significant only at the $100 \mathrm{~ms} \operatorname{SOA}(\mathrm{F}(1,124)=$ 11.72, $\mathrm{p}<0.01)$. At this cue-target interval, 22 of the 32 participants were faster to detect valid targets. At the $500 \mathrm{~ms}$ SOA, 16/32 participants showed this effect, whereas by $1000 \mathrm{~ms}$ only $11 / 32$ participants were faster to detect validly cued targets.

\section{Discussion}

The results of this experiment provide some preliminary evidence for reflexive spatial orienting to centrally located head precues. Participants were faster to detect the presence of a visual target when it was immediately preceded by a valid head cue than when it was preceded 
by an invalid head cue. This effect occurred despite the facts that participants were instructed to ignore the cue, and that these cues were not predictive of the exact location of the target. Moreover, this cueing effect was rapid, appearing $100 \mathrm{~ms}$ after the onset of the head cue, and short lived (absent for the longer SOA's). These observations are consistent with an exogenous, or stimulus driven, orienting mechanism, normally engaged by abrupt visual onsets in the periphery of the visual field (e.g., Yantis \& Jonides, 1984), but not thought to be engaged by central cues such as arrows, or the head stimuli used in this experiment. These cues would normally be expected to engage only the strategic or endogenous orienting mechanism (e.g., Müller \& Rabbitt, 1989).

A possible argument against an exogenous orienting explanation for the effects in this experiment is that despite the fact that the cues were completely uninformative regarding the exact location of the target, they nevertheless predicted the actual axis on which the target would appear with a $100 \%$ reliability. Thus there was an incentive for participants to make some kind of strategic interpretation of the cue in order to reduce the uncertainty of the likely target location. Despite the fact that the effects in this experiment seem to have occurred too rapidly to suggest the operation of such an endogenous mechanism, a further study was conducted in order to exclude this possibility.

\section{Experiment 2}

In Experiment 2 the cue validity was reduced to $25 \%$ so that following any cue, targets were equally likely to appear in any of the four possible locations. The head cues were therefore completely uninformative regarding the likely location of the target stimulus. Participants no longer had any incentive to attend to the cue and as a result, any advantage for detecting valid targets over invalid targets will presumably reflect the operation of an exogenous reflexive orienting mechanism. 


\section{Method}

Participants. These were fifteen undergraduates who were recruited through advertisements. All had normal, or corrected to normal vision.

Apparatus and Materials. Stimuli were presented on a Macintosh Quadra 800, again with a 14 inch monitor. Otherwise the materials were identical to those used in Experiment 1.

Design. As in Experiment 1 the materials were tested in a within subjects design with three factors: Cue Condition (valid or invalid), SOA (100 ms and $1000 \mathrm{~ms}$ ) and Cue Axis (vertical or horizontal). The $500 \mathrm{~ms}$ SOA condition included in Experiment 1 was dropped to increase the power of the design.

Procedure. Trials began with the appearance of a fixation cross displayed in the centre of the screen for $1500 \mathrm{~ms}$. Participants were asked to fixate on this cross and to maintain fixation in the centre of the screen throughout each trial in the experiment. Following the cross, one of the head cues was presented for a duration of $50 \mathrm{~ms}$. After a delay of either $50 \mathrm{~ms}$ (for the $100 \mathrm{~ms}$ SOA condition) or $950 \mathrm{~ms}$ (for the $1000 \mathrm{~ms}$ SOA condition) the target letter was presented at one of the four target locations, along with the fixation cross in the centre of the screen. This display remained on the screen until the participant had responded by pressing the space bar on the keyboard. The screen then remained blank for $1000 \mathrm{~ms}$ before the next trial began.

Unlike the previous experiment, targets in Experiment 2 did not necessarily appear on the same axis as the cue. In fact, following any cue, the target was equally likely to appear in any one of the four possible target locations. Thus, in only $25 \%$ of the trials did the cue correctly predict the location of the target, and in the remaining $75 \%$ of trials, the cue did not correspond with the location of the target. Participants were told about these contingencies and were thus asked to ignore the heads.

Each participant received 24 valid, 72 invalid, and 24 catch trials in each SOA condition, giving a total of 240 trials. These were divided into three identical blocks of 80 trials. Prior to the 
experimental block, participants completed a practice block of 20 trials containing 4 valid, 12 invalid, and 4 catch trials. A set of four practice trials also preceded each experimental block. Trials were presented in a random order within each of these blocks.

\section{Results}

As there were more trials in the invalid than in the valid condition of this experiment, mean rather than median RT's were computed for each participant (see Miller, 1988). RT's of less than $150 \mathrm{~ms}$ or greater than $1500 \mathrm{~ms}$ were excluded from the subsequent analysis. The percentage of trials eliminated by this procedure was $0.1 \%$. The resulting interparticipant mean RT's and standard deviations for each condition are summarised in Table 2. This table shows a clear $19 \mathrm{~ms}$ effect of cue condition at $100 \mathrm{~ms}$ SOA, but only a $2 \mathrm{~ms}$ effect at $1000 \mathrm{~ms}$ SOA. Responses also tended to be slower for the longer SOA condition. On average, participants made $1.67 \%$ catch trial errors.

Table 2

Mean Reaction Times (RTs; in milliseconds) and Standard Deviations for Detecting Targets Cued by Horizontal and Vertical Valid and Invalid Heads in Experiment 2.

Stimulus Onset Asynchrony

\begin{tabular}{|c|c|c|c|c|}
\hline \multirow[b]{2}{*}{ Cue Axis } & \multicolumn{2}{|c|}{$100 \mathrm{~ms}$} & \multicolumn{2}{|c|}{$1000 \mathrm{~ms}$} \\
\hline & Valid Cue & Invalid Cue & Valid Cue & Invalid Cue \\
\hline \multicolumn{5}{|l|}{ Horizontal } \\
\hline $\mathrm{R}^{\prime}$ & 373 & 389 & 394 & 399 \\
\hline SI & 39 & 42 & 55 & 55 \\
\hline \multicolumn{5}{|l|}{ Vertical } \\
\hline $\mathrm{R}^{\prime}$ & 369 & 390 & 400 & 398 \\
\hline SI & 38 & 34 & 59 & 49 \\
\hline \multicolumn{5}{|l|}{ Average } \\
\hline $\mathrm{R}^{\prime}$ & 371 & 390 & 397 & 399 \\
\hline SI & 38 & 37 & 56 & 51 \\
\hline
\end{tabular}


A 2 (Cue Condition) x 2 (SOA) x 2 (Cue Axis) ANOVA conducted on participants mean RT's supported the above observations. There were main effects of Cue Condition $(\mathrm{F}(1,14)=$ 11.17, $\mathrm{p}<0.01)$ and $\operatorname{SOA}(\mathrm{F}(1,14)=6.02, \mathrm{p}<0.05)$ as well as a significant interaction between these two factors $(\mathrm{F}(1,14)=8.49, \mathrm{p}<0.01)$. No other effects or interactions approached significance (p's $>0.1$ ). Simple main effects analysis of the Cue Condition x SOA interaction indicated a significant effect of cue condition at $100 \mathrm{~ms} \operatorname{SOA}(\mathrm{F}(1,28)=19.51, \mathrm{p}<0.001)$, but no effect at the longer $1000 \mathrm{~ms}$ SOA ( $>0.1$ ). AT $100 \mathrm{~ms}$ SOA, fourteen of the fifteen participants showed this effect whereas by $1000 \mathrm{~ms}$, only seven participants showed an advantage for valid trials.

\section{Discussion}

The findings of this experiment corroborate those of Experiment 1. As before there was an advantage for valid over invalid targets when the targets appeared $100 \mathrm{~ms}$ after the onset of the cue but no such advantage in the longer SOA condition. Moreover, in this experiment the cueing effect was obtained despite the fact that the head precues were now completely uninformative regarding both the axis and the likely location of the target. This observation, coupled with the fact that the cues exerted their effects within $100 \mathrm{~ms}$ of their onset, further suggests the operation of an exogenous orienting mechanism which is engaged reflexively by head direction precues.

The findings of Experiments 1 and 2 represent an unusual case of an exogenous orienting response engaged by a central symbolic cue. As noted earlier, central cues were hitherto thought to engage only the slower endogenous orienting mechanism. Orienting to these cues was thought to be under voluntary control and could therefore be suppressed by instructing participants to ignore the cues, or by arming them with knowledge that the cues were not predictive of the likely location of the target. However, neither of these manipulations were successful in preventing an orienting response to the head cues in Experiments 1 and 2. It seems that these central symbolic cues provoke a purely stimulus driven or exogenous response which is beyond the strategic control of the observer. 


\section{Experiment 3}

Experiment 3 was designed to further examine whether the orienting effect obtained at 100 ms SOA's in Experiments 1 and 2 is purely stimulus driven and reflexive, or whether it can be influenced by participants' expectancy. As before, target detection latencies were measured for cued and uncued targets, however in this experiment the cued location was three times as likely to contain a target as any one of the three uncued locations and participants were actually asked to concentrate on the cues. As a result, the head cues were now relatively informative regarding the likely location of the target. If the advantage for target detection at cued over uncued locations at $100 \mathrm{~ms}$ SOAs in Experiments 1 and 2 is the result of a purely stimulus-driven or exogenous orienting process, then participants' knowledge or intentions would not be expected to exert any influence over the corresponding effect in this experiment. Thus, we expected to obtain a cueing effect at $100 \mathrm{~ms}$ SOA of similar magnitude to those found in Experiments 1 and 2. On the other hand, we would now expect to observe an effect at the longer $1000 \mathrm{~ms}$ SOA as this should give participants sufficient time to effect a strategic or endogenous shift of attention contingent on the direction of the cue.

\section{Method}

Participants. These were sixteen undergraduate and post-graduate students at the University of Stirling, none of whom had participated in the earlier experiments. All had normal, or corrected-to-normal vision.

Apparatus and Materials. These were identical to those used in Experiment 2.

Design \& Procedure. As in the previous experiments there were three within subjects factors: Cue Condition (valid or invalid cues), SOA (100 ms or $1000 \mathrm{~ms}$ ) and Cue Axis (horizontal or vertical). However, in this experiment half of the trials were valid and half invalid (50\% cue validity). Targets were presented with equal frequency at each of the four locations on the screen so that on invalid trials, the target was equally likely to appear in any of the three uncued 
locations. Consequently, the cued location was three times as likely to contain a target as any one of the three uncued locations. Each participant completed two blocks of 96 experimental trials. These comprised twelve valid and twelve invalid trials at each of the SOA conditions as well as 48 catch trials. The participants were told that although the head precues did not always predict the location of the target, on average their performance would be improved by attending to these stimuli. However, it was stressed that they should maintain fixation on the central cross.

\section{Results}

Median RTs for each participant were calculated as in Experiment 1. The mean of participant's median RTs and standard deviations for all conditions appear in Table 3. The average rate of catch trial errors made by participants was $0.46 \%$.

Table 3

Means of Median Reaction Times (RTs; in milliseconds) and Standard Deviations for Detecting Targets Cued by Horizontal and Vertical Valid and Invalid Heads in Experiment 3.

Stimulus Onset Asynchrony

\begin{tabular}{rccccc}
\cline { 3 - 5 } Cue Axis & \multicolumn{2}{c}{$100 \mathrm{~ms}$} & \multicolumn{2}{c}{$1000 \mathrm{~ms}$} \\
\cline { 2 - 5 } & & Valid Cue & Invalid Cue & Valid Cue & Invalid Cue \\
\hline \multirow{2}{*}{ Horizontal } & & & & \\
& RT & 360 & 383 & 404 & 420 \\
& SD & 41 & 42 & 62 & 63 \\
Vertical & & & & & 410 \\
& RT & 363 & 378 & 60 & 57 \\
& SD & 40 & 45 & & 421 \\
Average & & & & 407 & 59 \\
& RT & 362 & 381 & 60 & \\
& SD & 40 & 43 & & \\
\hline
\end{tabular}

An inspection of Table 3 reveals a $19 \mathrm{~ms}$ advantage for valid over invalid cues at $100 \mathrm{~ms}$ SOA, and a $14 \mathrm{~ms}$ advantage at $1000 \mathrm{~ms}$ SOA. Target detection latencies are also faster for shorter SOA's. These observations were supported by a 2 (Cue Condition) x 2 (SOA) x 2 (Cue 
Axis) ANOVA conducted on participants' median RT's in each condition. This analysis revealed a main effect of SOA $(\mathrm{F}(1,15)=24.16, \mathrm{p}<0.001)$ with participants faster to respond at the shortest SOA. There was also a main effect of cue condition $(F(1,15)=24.22, p<0.001)$ with faster responses on valid trials. This effect was evident for fourteen participants at $100 \mathrm{~ms} \mathrm{SOA}$ and twelve participants at the longer cue-target interval. Neither the interaction between validity and SOA, nor any of the other effects or interactions reached significance (p's >0.1).

\section{Discussion}

In Experiments 1 and 2 uninformative head stimuli produced reliable precueing effects within $100 \mathrm{~ms}$ of the onset of the cue, but no effects at longer SOA's. By increasing the informativeness of the head cues in Experiment 3, a precueing effect was induced at $1000 \mathrm{~ms}$ SOA, in addition to an effect at the shorter cue-target interval. These findings are consistent with the operation of an exogenous orienting mechanism at the shorter SOA and a slower acting endogenous response producing the cueing effect at the $1000 \mathrm{~ms}$ SOA.

A comparison of the results of this experiment with those of Experiments 1 and 2 suggests that manipulation of the informativeness of the cue influenced the cueing effect at $1000 \mathrm{~ms}$ but had little influence on the magnitude of cueing effect at the $100 \mathrm{~ms}$ cue-target interval (13 ms, 19 ms and $19 \mathrm{~ms}$ in Experiments 1, 2 and 4 respectively). This dissociation suggests that head precues are able to engage both exogenous and endogenous orienting mechanisms. Exogenous orienting benefits target detection at shorter SOAs, whereas the endogenous mechanism produces an advantage for detection of valid over invalid targets at longer cue-target intervals.

The cueing effects obtained at $100 \mathrm{~ms}$ SOA's in Experiments 1-3 have been interpreted in terms of an exogenous orienting response which is triggered by the head orientation and/or gaze direction of the cue. However, another possibility is that the visual orienting is elicited by some physical property of the cue which is confounded with the cue's gaze or head direction. One suggestion is that participants automatically orient their attention toward the more visually complex or salient components of the cueing stimuli. For example, with cues on the horizontal 
axis, the more visually "interesting" aspects of the stimuli are provided by the occluding contour of the face's profile, which, of course, is always perfectly correlated with the direction of the head/gaze cue. Detection performance for targets at the cued location may then be enhanced because this initial shift brings the target closer to the focus of attention, and target detection time has been shown to improve as the distance between the focus of attention and the target decreases (e.g., Downing \& Pinker, 1985). However, whilst this explanation of the cueing effects may hold for the horizontal cues, it does not seem as compelling for head cues oriented on the vertical axis where the asymmetry in visual complexity is arguably not as striking. Indeed, the results of Experiments 1-3 indicate that, despite the absence of any obvious asymmetries, vertical cues produce identical cueing effects to cues on the horizontal axis.

Rather than orient attention toward regions of visual complexity, it may be that the advantage for target detection at cued locations is caused by participants tendency to shift their attention toward certain facial "features" of the cues. Studies examining eye-movements in the inspection of faces (Yarbus, 1967), or during various face matching tasks (Walker-Smith, Gale \& Findlay, 1977) have shown that viewers tend to fixate their gaze on the eye, mouth and nose regions of the face. If participants adopt this strategy in the present experiments, their attention will be oriented somewhat automatically toward the target stimulus on valid trials, enhancing the target detection time as described above.

To summarise, the preceding discussion has raised the possibility that the cueing effects obtained at $100 \mathrm{~ms}$ SOA's in Experiments 1-3 are due to participants tendency to orient their attention toward certain aspects of the cueing stimulus which are perfectly correlated with the cue's direction of attention. The suggestion is either that attention is attracted to regions of visual complexity within a stimulus, or that attention is automatically directed toward certain facial features, in particular the eyes, mouth and nose. In either case, attention will be focussed on a region closer to the target on valid trials, and further from the target on invalid trials. This will tend to produce a cueing effect, not because of an attentional shift made on the basis of the gaze 
or head direction, but because of the way visual attention is allocated to the cues themselves. The next experiment was designed to explore these issues.

\section{Experiment 4}

In Experiment 4 the cueing stimuli used in the previous experiments were each rotated about $180^{\circ}$ to produce a set of inverted head cues. Numerous studies have demonstrated that inversion severely disrupts various aspects of face processing (e.g., Bruce \& Langton, 1994; Diamond \& Carey, 1986; Valentine \& Bruce, 1986; Yin, 1969). For instance, Yin (1969) showed that recognition memory for upright faces was better than that for pictures of houses, aeroplanes, or schematic men-in-motion, but when all these materials were inverted, performance on the faces became worse than that on the other pictures.

In addition to face recognition performance, inversion also seems to disrupt the discriminatation of gaze direction. As described in the Introduction, the context of the face (e.g., upright or inverted) has been shown to influence participants' sensitivity to eye-gaze direction, even when the eyes remain upright within the context of, say, an inverted face (Jenkins, in prep; Vecera \& Johnson, 1995). However, the inversion of the eyes within the context of an upright face also compromises sensitivity to their direction of gaze (Jenkins, in prep). These findings seem to suggest that inversion disrupts performance on gaze discrimination tasks because of a combination of the rotatation of the face context and the rotation of the eyes themselves.

If the cueing effects observed at shorter SOA's in Experiments 1-3 were the result of the perception of gaze/head orientation triggering an exogenous orienting response, then the effect should be reduced or eliminated by the inversion of the head cues. On the other hand, if the cueing effects were due to a tendency to orient attention toward regions of visual complexity or to particular face features, then because these regions are unchanged by inversion, the cueing effects should persist. Indeed, inversion might be expected to actually enhance the processing of individual face features perhaps facilitating any visual orienting to these regions. This is because inversion is widely considered to disrupt the holistic or configural processing which normally 
operates on upright faces (e.g., Yin, 1969), allowing the components of the inverted face to be processed independently.

\section{Method}

Participants. These were fifteen undergraduate and post-graduate students at the University of Stirling, none of whom had participated in the earlier experiments. All had normal, or corrected-to-normal vision.

Apparatus and Materials. The head stimuli used in Experiments 1-3 were each rotated by $180^{\circ}$ to produce a set of four inverted heads which were used as the cueing stimuli in this experiment. Otherwise the apparatus and materials were the same as those used in Experiments $1-3$.

Design \& Procedure. The design and procedure were identical to those used in Experiment 2.

\section{Results}

Mean RTs were computed for each participant under each of the experimental conditions as in Experiment 2. Again RTs greater than $1500 \mathrm{~ms}$ or less than $150 \mathrm{~ms}$ were removed from the analysis. This trimming procedure eliminated just $0.17 \%$ of the data. The resulting interparticipant mean RT's and standard deviations for each condition are summarised in Table 4. Overall, valid trials enjoy a small $8 \mathrm{~ms}$ advantage over invalid trials at $100 \mathrm{~ms} \mathrm{SOA}$, but the effect of cue condition is reduced to $-4 \mathrm{~ms}$ at the longer $1000 \mathrm{~ms}$ SOA. The average rate of catch trial errors was $1.94 \%$. 
Table 4

Mean Reaction Times (RTs; in milliseconds) and Standard Deviations for Detecting Targets Cued by Horizontal and Vertical Valid and Invalid Heads in Experiment 4.

Stimulus Onset Asynchrony

\begin{tabular}{|c|c|c|c|c|}
\hline \multirow[b]{2}{*}{ Cue Axis } & \multicolumn{2}{|c|}{$100 \mathrm{~ms}$} & \multicolumn{2}{|c|}{$1000 \mathrm{~ms}$} \\
\hline & Valid Cue & Invalid Cue & Valid Cue & Invalid Cue \\
\hline \multicolumn{5}{|l|}{ Horizontal } \\
\hline RT & 356 & 367 & 406 & 413 \\
\hline $\mathrm{SD}$ & 49 & 51 & 46 & 55 \\
\hline \multicolumn{5}{|l|}{ Vertical } \\
\hline RT & 362 & 366 & 425 & 410 \\
\hline SD & 42 & 48 & 52 & 52 \\
\hline \multicolumn{5}{|l|}{ Average } \\
\hline RT & 359 & 367 & 416 & 412 \\
\hline SD & 45 & 49 & 49 & 53 \\
\hline
\end{tabular}

A three-way within subjects ANOVA (Cue Condition x SOA x Cue Axis) conducted on the RT data found a main effect of SOA $(\mathrm{F}(1,14)=45.15, \mathrm{p}<0.001)$, with participants responding faster at the shorter cue-target interval as before. The main effect of SOA was qualified by a significant Cue Condition x SOA interaction $(\mathrm{F}(1,14)=4.71, \mathrm{p}<0.05)$. Further analysis of this interaction confirmed the observations made earlier. Valid cues produced a marginally significant target detection advantage over invalid cues at the shorter $100 \mathrm{~ms} \operatorname{SOA}(\mathrm{F}(1,28)=$ $3.85, \mathrm{p}=0.06)$ but not in the longer $1000 \mathrm{~ms}$ SOA condition $(\mathrm{p}>0.1)$. However, the cueing effect was further qualified by a significant interaction between cue condition and cue axis ( $\mathrm{F}(1$, 14) $=8.21, \mathrm{p}<0.05)$. Tests of simple main effects showed that the effect of cue condition was significant for horizontal cues $(\mathrm{F}(1,28)=5.44, \mathrm{p}<0.05)$ but not for cues on the vertical axis $(\mathrm{p}>$ 0.1). The overall ANOVA also yielded a marginally significant main effect of cue axis $(F(1,14)$ $=4.18, \mathrm{p}=0.06$ ) with RTs to targets preceded by horizontally oriented cues tending to be faster than RTs to targets preceded by vertical cues. No other effects or interactions reached significance (p's >0.1). 


\section{Discussion}

In Experiments 1 and 2, upright head cues on both the horizontal and vertcal axes produced reliable cueing effects at short, $100 \mathrm{~ms}$ cue-target intervals, but no such effects at $1000 \mathrm{~ms}$ SOA's. The inverted head cues used in Experiment 4 also produced a marginally significant cueing effect at short SOA's but, in contrast to the previous findings, cueing effects were resticted to inverted heads appearing on the horizontal axis. No cueing effects were obtained for cues on the vertical axis.

Several observations can be made concerning the effects of inverting the head cues by comparing the findings of Experiment 4 directly with those of Experiment 2 which had similar power and an identical experimental design. First, it is clear that the $8 \mathrm{~ms}$ cueing effect produced by inverted heads at $100 \mathrm{~ms} \mathrm{SOA}$ is much smaller in magnitude than the $19 \mathrm{~ms}$ effect obtained with upright heads in in the comparable SOA condition of Experiment 2. In order to compare the cueing effects obtained with upright and inverted heads, the RT data from the $100 \mathrm{~ms}$ SOA conditions of Experiments 2 (upright heads) and 4 (inverted heads) were entered into a mixed ANOVA with orientation (upright vs. inverted cues) as a between-subjects factor and cue condition (valid vs. invalid cues) as a within-subjects factor. This analysis yielded a significant main effect of condition $(\mathrm{F}(1,28)=28.09, \mathrm{p}<0.001)$ and, importantly, a significant interaction between cue condition and orientation $(F(1,28)=6.45, \mathrm{p}<0.05)$. Tests of simple main effects confrimed that the effect of cue condition was significant for upright heads $(F(1,28)=30.71, p<$ $0.001)$, but only marginally significant for inverted heads $(\mathrm{F}(1,28)=3.82, \mathrm{p}=0.06)$. This analysis therefore provides statistical support for the observation that inversion severely reduces, but does not completely eliminate the cueing effect. Thus it is suggested that the rotation of the head cues disrupts the computation of the gaze and/or head direction of the cue, and that this, in turn, either retards or eliminates the normal visual orienting response.

A second observation from a comparison of the cueing effects obtained with upright and inverted heads is that inverted head cues exert a qualitatively different form of cueing compared 
with upright heads. In both Experiments 1 and 2 the cueing effects produced by upright heads were as large for both vertical and horizontal cues. However, in Experiment 4 the effect of cue condition was only evident for horizontal cues, and completely absent for vertical cues. This finding clearly rules out the possibility that the upright vertical cues produce their effects because participants tend to direct their attention toward certain facial features or to regions of visual complexity within the cues. Rotation of the vertical cues completely eliminated any detection advantage for valid targets despite the fact that, in this case, the asymmetry in visual complexity (if any exists), and the location of the features within the face are still correlated with the location of target.

The idea that participants orient attention toward certain facial features, or to visually interesting regions should not, however, be completely ruled out. It may well be this tendency which produced the cueing effect observed with the horizontally oriented cues. It is suggested that, as described above, inversion disrupts the processing of gaze/head orientation which in turn eliminates the corresponding visual orienting response. The small detection advantage for targets preceded by valid horizontal cues in Experiment 4 is therefore considered to be the result of the way in which participants' allocate attention to the cues themselves. No such residual cueing effects were noted for vertical heads as the visual asymmetries in these cues are in no way as salient as those in the corresponding horizontal cues and, as such, are not sufficient to attract participants' visual attention. Moreover, it seems unlikely that attention is allocated specifically to features within the face as, if this were the case, some kind of cueing effect would have been observed with cues on the vertical axis. It therefore seems more likely that the small cueing effects noted in Experiment 4 are the result of an automatic tendency to orient attention toward visually complex areas rather than to the face features themselves.

In summary, it is argued that the effect of inverting the head cues in Experiment 4 was to eliminate the cueing effect usually made in response to another's direction of attention. The small effects which were observed with cues on the horizontal axis were considered to be a consequence of the way in which participants allocate attention to these stimuli, rather than a 
visual orienting response triggered by their direction of attention. These findings make it unlikely that the $100 \mathrm{~ms}$ SOA cueing effects observed in Experiments 1-3 were caused exclusively by the physical properties of the cues. Rather, they suggest that the effects were primarily the result of attentional shifts stimulated by the social signals contained within the face cues. In fact, recent independent work by other groups (Driver, Davis, Kidd, Maxwell, Ricciardelli \& Baron-Cohen, submitted; Friesen \& Kingstone, submitted) reinforce the claim that central social cues are able to trigger a reflexive orienting of attention. For instance, in Driver et al.'s experiments a change in gaze direction with no change in head orientation served as a central, uninformative cue to the location of a target stimulus in a letter discrimination task. Similar to the present findings, Driver et al. showed that performance was faster when the letter stimuli appeared at cued as opposed to uncued locations. Friesen and Kingstone obtained similar results using schematic gaze cues in detection, discrimination and localisation tasks. In both the Driver et al. and Friesen and Kingstone studies, performance was affected by the prior presentation of a social cue which had no obvious visual asymmetries. These cueing effects could not, therefore, be caused by a tendency to orient attention toward areas of visual complexity, toward the eye region, or to other particular facial features. Instead, these findings, coupled with the results of the present experiments, suggest that central social signals such as eye gaze and head orientation are indeed able to trigger a reflexive visuospatial orienting of attention.

\section{General Discussion}

The aim of the experiments reported in this article was to explore the hypothesis that social attention cues are able to exert a reflexive influence on the attentional orientation of the observer. Experiment 1 revealed a significant precueing effect with $100 \mathrm{~ms}$ SOA between the head precueing stimuli and target letters. Thus, at the shorter cue-target intervals, participants were faster to detect the presence of a target when the location of this stimulus had previously been indicated by the direction of a to-be-ignored head precue than when the precue indicated a different location. However, the advantage for target detection at cued over uncued locations was relatively short-lived as there was no evidence of a cueing effect at longer cue-target durations. 
These findings were corroborated by those of Experiment 2. Once again a cueing effect was obtained at a short but not a long SOA, even when the precue was completely uninformative regarding the likely location of the target. Experiment 3 was designed to pursue the hypothesis that the cueing effects triggered by the head cues were purely stimulus-driven or reflexive in nature. Participants' expectancies were manipulated by making the head cues relatively informative regarding the likely location of the target. Compared with Experiments 1 and 2, this manipulation did not influence the magnitude of the $100 \mathrm{~ms}$ SOA cueing effect suggesting that it is indeed the result of a reflexive orienting of attention. However, an effect was introduced at $1000 \mathrm{~ms}$ SOA, presumably reflecting the operation of the voluntary endogenous orienting mechanism. Finally, inversion of the head cues (Experiment 4) produced a pattern of results which differed both quantitatively and qualitatively from those obtained in Experiment 2, making it unlikely that the cueing effects observed at short SOA's in the preceding experiments were exclusively a consequence of the physical properties of the stimuli (e.g., visual complexity or salience), or a tendency on behalf of participants to direct attention toward particular facial features.

Together, the findings of Experiments 1-4 demonstrate that another's head orientation can induce spatial orienting in an observer despite the facts that the head cues were ignored and completely irrelevant to the experimental task (Experiments 1 and 2). In many respects these attention shifts resemble conventional exogenous or reflexive orienting; the effects occurred at short, but not long SOA's, they were observed even when the cue provided no information regarding the likely location of the target, and were uninfluenced by participants' intentions or expectancy.

However, one observation which is inconsistent with an account based on exogenous orienting is the absence of inhibition of return (IOR) at the longer SOA conditions of Experiments 1 and 2. In these experiments participants' attention is thought to be deployed reflexively toward the cued location, benefiting detection of any immediately presented target. However, at longer SOA's, participants have ample time to withdraw their attention from the 
cued location and return it endogenously to fixation before the appearance of any target. Numerous studies have shown that, under these conditions, some kind of inhibitory process retards the detection of any target which subsequently appears at the recently attended location (e.g., Maylor \& Hockey, 1985; Posner \& Cohen, 1984; Rafal et al., 1991). Posner \& Cohen (1984) argued that this inhibition of return effect favours novelty in visual scanning, and showed that it could be obtained at SOA's greater than $300 \mathrm{~ms}$. In view of this, an IOR effect might have been expected at the longer cue-target intervals in Experiments 1 and 2.

Posner and Cohen (1984) initially attributed IOR to a process which inhibits reorienting to recently stimulated locations. On this view, it is the change in light energy produced by the peripheral cue which causes the effect, rather than the actual orienting mechanism per se. Because the central head precues used in Experiment 1 and 2 do not produce a change in light energy at the locations they indicate, according to Posner and Cohen's (1984) argument, there is no reason to suspect any inhibitory effects for targets which might then appear at these positions. Subsequently however, Posner et al. (1985) showed that IOR could be observed even in the absence of peripheral stimulation. In their experiments, centrally located arrow cues directed participants to make a saccade to some eccentric location which already contained a small digit. Participants had to read this digit before returning their eyes to the initial fixation point. IOR was obtained for subsequent detection of targets which appeared at the location to which the initial saccade had been made. More recently, Rafal et al. (1989) showed that central arrow cues only produce IOR effects when participants either plan or actually make a saccade to the cued location. In their view, IOR arises from activation of the occulomotor system rather than from sensory processes or from the orienting of covert attention per se. To summarise, the evidence suggests that peripheral cues always produce IOR either through automatic preparation and/or execution of eye movements, or because of peripheral stimulation at the site of the cue. Central cues, on the other hand, only produce IOR if eye-movements are endogenously planned and/or executed. In the present studies, participants were instructed to remain fixated in the centre of the display throughout each trial in the experiment. They were neither asked to make, nor plan any 
eye movements. Therefore, following Rafal et al. (1989), the absence of IOR in the present studies may be due to the fact that the occulomotor system was not endogenously primed to respond in the direction indicated by the head cues. In this respect the social cues were acting very much like the more traditional central cues used to explore endogenous orienting.

A second observation at variance with standard reflexive visual orienting is that the cueing effects in the present experiments were obtained with centrally located cues and eccentric targets. So for instance, the location of a valid cue did not correspond with the location of the subsequent target stimulus as is usually the case in demonstrations of reflexive orienting (e.g., Jonides, 1981; Posner \& Cohen, 1984). Indeed, it is remarkable that we have obtained any cueing effects at all given this spatial separation between cueing and target stimuli. In particular the appearance of the central head cue produces a substantial central transient which would tend to draw exogenous attention to the central location. This may well retard any subsequent orienting in the direction indicated by the head.

Thus, there are at least two properties of the cueing effects which are inconsistent with a standard reflexive orienting process. First, there was no evidence of inhibition of return at longer cue-target intervals in any of the experiments reported above, and second, the effects were not tied to the location of the cue. These observations are more indicative of endogenous than exogenous orienting. However, it is unlikely that the effects were caused by any purely voluntary shifting of attention for several reasons. First, the effects occurred despite the fact that participants were told at the beginning of the experiment that the cues did not predict the likely location of the target. Participants were therefore not required to learn the contingencies over the experimental session, and hence were given no incentive to deploy endogenous attention at any stage of the experiment. Second, participants in Experiments 1 and 2 were explicitly asked to ignore the cues because they were uninformative regarding the location of the target. Finally the effect was not influenced by the additional deployment of endogenous attention in Experiment 3 where participants were asked to attend to relatively informative head cues. 
The effects observed in the above experiments, whilst unlikely to be caused by an endogenous process, are nevertheless clearly somewhat different to conventional exogenous orienting. One possibility is the present findings are characteristic of a special kind of orienting response which is triggered exclusively by such social signals as gaze or head direction. In this case the absence of IOR, or indeed any other observations which are at variance with normal exogenous orienting, may actually be characteristic of this kind of "social" orienting response. Clearly this suggestion is one which requires further investigation. Future studies could compare the time courses of the cueing effects produced by non-social cues with those of social signals, or attempt to dissociate the putative social orienting response from the exogenous orienting mechanism.

Rather than postulating a special social orienting mechanism, it is perhaps more likely that the cueing effects observed in the present experiments are the result of a more general mechanism which is based on the associative learning between cue attributes and target events. Lambert and Sumich (1996) have recently presented some evidence for just such an attentional mechanism. In their experiments, Lambert and Sumich presented word cues to the left or right of participants' fixation. Unbeknownst to these participants, the semantic category of these words was predictive of the likely location of a subsequent target stimulus to which they were asked to make a simple RT response. Although the participants were unaware of the cue-target location relationship, they were nevertheless significantly faster to detect targets appearing in the cued, compared with the uncued locations. The spatial orienting producing this effect was suggested to be the result of an implicit or non-conscious learning of the association between attributes of the cue and target events.

It may be that the visual orienting observed in the present experiments is the result of a similar learning process. Presumably, experience has taught us, either implicitly or explicitly, that a change in another's attention direction is generally associated with the appearance of objects of interest or threat in the environment. Moreover, this association will be built up from a lifetime's worth of experience, rather than the learning of statistical regularities over just a few 
blocks of trials in the course of an experimental session. According to Lambert and Sumich's (1996) argument, this learning would result in the development of a spatial orienting response to incidental gaze or head direction cues which may be so overlearned that participants have difficulty surpressing it at will. Indeed, it may well be that this learning process strengthens the link between Perrett and Emery's (1994) putative Direction of Attention Detector and the attentional orienting mechanisms of the parietal cortex (Harries \& Perrett, 1991).

Corkum and Moore (1995) have suggested that learning is indeed a possible route for the acquisition of the gaze following response observed in infants. They showed that spontaneous gaze following was not evident in children younger than 10-11 months, but that a considerable number of 8-11 month old children were nonetheless able to learn, through conditioning, to make head turn responses in line with an adult's direction of gaze. However, others have shown that these kinds of responses might be available very much earlier than Corkum and Moore (1995) propose. For instance, studies by Scaife and Bruner (1979) and Butterworth and Jarrett (1991) suggest that some kind of primitive response is available at two months of age. In line with this, Hood, Willen and Driver (Driver, personal communication) found, using a cueing paradigm, that infants as young as ten weeks were faster to saccade toward a peripheral probe which had just been cued by the gaze direction of a centrally located face cue. This latter study represents a significant advancement in the study of joint attention in infancy as the measurement of saccade latency would seem to be a more sensitive measure of joint attention than are instances of head turning or changes in eye gaze direction. Measuring young childrens' rapid and reflexive orienting responses in this way might allow more accurate plotting of the ontogenesis of joint visual attention behaviours in infancy. However, whether the orienting effects we have observed are the result of a learning process, or some kind of innate mechanism, as implied by BaronCohen (1995), is clearly a difficult issue to resolve.

In summary, the results of the experiments reported here are interpreted in terms of an overlearned reflexive orienting response which is triggered by the perception of head orientation. This account is, therefore, based on exogenous, rather than endogenous control of attention. 
However, what of the distinction between overt and covert orienting also made by researchers of visual attention? The fact that participants were strongly encouraged to remain fixated throughout each experiment, and were reminded to do so between each block of trials would seem to favour an explanation based on covert orienting. However, it is equally possible that the head cues produce an overt reflexive saccade in the cued direction so as to foveate the potential location of the target. The cueing effects would then represent an RT advantage on valid trials because of increased foveal sensitivity at the cued locations. A third possibility is that both overt and covert shifts could have occurred. Unfortunately, because eye movements were not explicitly monitored in these experiments it is impossible to distinguish between these possibilities. However, this in no way weakens the conclusion that some form of reflexive orienting must have occurred in order to produce the cueing effects we have observed. Future research aims to determine the extent to which these effects are caused by covert orienting, overt orienting, or both.

\section{Conclusion}

The experiments reported in this paper clearly establish that face stimuli, indicating direction by virtue of their head and eye position, produce a reflexive orienting response on behalf of the observer. Of course, it remains to be seen whether these effects are based on an orienting response to the head, the direction of gaze, or a combination of the two. Perrett's putative Direction of Attention Detector (Perrett et al., 1992; Perrett \& Emery, 1994) is sensitive to both of these cues, in addition to information carried by body posture. On this basis we might expect head, eye and body cues presented in isolation to induce identical reflexive orienting effects. As described earlier, both Driver et al. (submitted) and Friesen and Kingstone (submitted), have recently shown that that eye-gaze direction alone does indeed trigger this same automatic visuospatial orienting response in adult human observers. In addition, our own work on the processing of gestural and verbal information in comprehension has hinted that pointing gestures also serve as an important cue to social attention direction, and may thus exert similar orienting effects on an observer's visual attention (Langton, 1996; Langton, O’Malley \& Bruce, 1996). 
Thus, it may be that a number of important social signals are all capable of triggering the kind of attentional shifts observed in the present studies. One question, however, concerns how the system will respond to multiple, conflicting cues. Is information from the various signals combined or integrated by something akin to Perrett and Emery's (1994) Direction of Attention Detector before the attentional shift is programmed and executed? How might this integration process proceed? In future work the intention is to continue to use some of the techniques and ideas favoured by experimental cognitive psychologists in order to explore these particular questions, and more generally, to study various aspects of social cognition within an information processing framework. For now the hope is that the present experiments have illustrated the fruitfulness of this line of enquiry. 


\section{References}

Baron-Cohen, S. (1994). How to build a baby that can read minds: Cognitive mechanisms in mindreading. Cahiers de Psychologie Cognitive, 13, 513-552.

Baron-Cohen, S. (1995a). The Eye Direction Detector (EDD) and the Shared Attention Mechanism (SAM): Two cases for evolutionary psychology. In C. Moore \& P. Dunham (Eds.), Joint attention: Its origins and role in development (pp. 41-59). Hillsdale, NJ: Lawrence Erlbaum Associates.

Baron-Cohen, S. (1995b). Mindblindness: An essay on autism and theory of mind. Cambridge, MA: MIT Press/Bradford Books.

Briand, K. A., \& Klein, R. M. (1987). Is Posner's "beam" the same as Treisman's "glue"?: On the relation between visual orienting and feature integration theory. Journal of Experimental Psychology: Human Perception and Performance, 13, 228-241.

Bruce, V., \& Langton, S. (1994). The use of pigmentation and shading information in recognising the sex and identities of faces. Perception, 23, 803-822.

Butterworth, G., \& Jarrett, N. (1991). What minds have in common is space: Spatial mechanisms serving joint visual attention in infancy. British Journal of Developmental Psychology, 9, 5572.

Campbell, R., Heywood, C. A., Cowey, A., Regard, M., \& Landis, T. (1990). Sensitivity to eye gaze in prosopagnosic patients and monkeys with superior temporal sulcus ablation. Neuropsychologia, 28, 1123-1142.

Cheal, M., \& Lyon, D. R. (1991). Central and peripheral precueing of forced-choice discrimination. Quarterly Journal of Experimental Psychology, 43A, 859-880. 
Corkum, V., \& Moore, C. (1995). Development of joint visual attention in infants. In C. Moore \& P. J. Dunham (Eds.), Joint attention: Its origins and role in development (pp. 61-83). Hillsdale, NJ: Lawrence Erlbaum Associates.

Diamond, R., \& Carey, S. (1986). Why faces are and are not special: An effect of expertise. Journal of Experimental Psychology: General, 115, 107-117.

Downing, C. J., \& Pinker, S. (1985). The spatial structure of visual attention. In M. I. Posner \& O. S. Martin (Eds.), Attention and performance XI Hillsdale, NJ: Erlbaum.

Driver, J., Davis, G., Kidd, P., Maxwell, E., Ricciardelli, P., \& Baron-Cohen, S. (submitted). Shared attention and the social brain: Gaze perception triggers automatic visuospatial orienting in adults. Visual Cognition.

Erikson, C. W., \& Collins, J. F. (1969). Temporal course of selective attention. Journal of Experimental Psychology, 80, 254-261.

Fodor, J. A. (1983). Modularity of mind: An essay on faculty psychology. Cambridge, MA: MIT Press.

Friesen, C. K. \& Kingstone, A. (submitted). The eyes have it!: reflexive orienting is triggered by nonpredictive gaze. Psychonomic Bulletin and Review.

Harries, M. H., \& Perrett, D. I. (1991). Visual processing of faces in the temporal cortex: Physiological evidence for a modular organisation and possible anatomical correlates. Journal of Cognitive Neuroscience, 3, 9-24.

Heywood, C. A., \& Cowey, A. (1992). The role of the "face cell" area in the discrimination and recognition of faces by monkeys. Philosophical Transactions of the Royal Society of London, Series $B ., 335,31-38$. 
Jenkins, J. (in prep) Detecting social signals from the face. Unpublished Doctoral Dissertation, University of Stirling, Stirling, UK.

Jonides, J. (1981). Voluntary versus automatic control over the mind's eye's movement. In J. B. Long \& A. D. Baddeley (Eds.), Attention and Performance IX (pp. 187-203). Hillsdale, NJ: Lawrence Erlbaum Associates.

Kleinke, C. L. (1986). Gaze and eye contact: A research review. Psychological Bulletin, 100, 78100.

Lambert, A. J., \& Sumich, A. L. (1996). Spatial orienting controlled without awareness: A semantically based implicit learning effect. Quarterly Journal of Experimental Psychology, $49 A, 490-518$.

Langton, S. R. H. (1996) Interference between gestures and words. Unpublished Doctoral Dissertation, University of Nottingham, Nottingham, UK.

Langton, S. R. H., O'Malley, C., \& Bruce, V. (1996). Actions speak louder than words: Symmetrical cross-modal interference effects in the processing of verbal and gestural information. Journal of Experimental Psychology: Human Perception and Performance, 22., $1357-1375$.

Lempers, J. D. (1979). Young children's production and comprehension of nonverbal deictic behaviors. The Journal of Genetic Psychology, 135, 93-102.

Lupiáñez, J., Milán, E. G., Tornay, F. J., Madrid, E., \& Tudela, P. (1997). Does IOR occur in discrimination tasks? Yes, it does, but later. Perception \& Psychophysics, 59, 1241-1254.

Maylor, E. A., \& Hockey, R. (1985). Inhibitory component of externally controlled covert orienting in visual space. Journal of Experimental Psychology: Human Perception and Performance, 11, 777-787. 
Miller, J. O. (1988). A warning about median reaction time. Journal of Experimental Psychology: Human Perception and Performance, 14, 539-543.

Müller, H. J., \& Findlay, J. M. (1988). The effects of visual attention on peripheral discrimination thresholds in single and multiple element displays. Acta Psychologica, 69, 129155.

Müller, H. J., \& Rabbitt, P. M. A. (1989). Reflexive and voluntary orienting of visual attention: Time course of activation and resistance to interruption. Journal of Experimental Psychology: Human Perception and Performance, 15, 315-330.

Perrett, D. I., \& Emery, N. J. (1994). Understanding the intentions of others from visual signals: Neurophysiological evidence. Cahiers de Psychologie Cognitive, 13, 683-694.

Perrett, D. I., Hietanen, J. K., Oram, M. W., \& Benson, P. J. (1992). Organisation and functions of cells responsive to faces in the temporal cortex. Philosophical Transactions of the Royal Society of London, Series B., 335, 23-30.

Perrett, D. I., Mistlin, A. J., Chitty, A. J., Harries, M., Newcombe, F., \& de Haan, E. (1988). Neuronal mechanisms of face perception and their pathology. In C. Kennard \& F. Clifford Rose (Eds.), Physiological aspects of clinical neuro-ophthalmology (pp. 137-154). London: Chapman \& Hall.

Posner, M. I. (1980). Orienting of attention. Quarterly Journal of Experimental Psychology, 32, $3-25$.

Posner, M. I., \& Cohen, Y. (1984). Components of visual orienting. In H. Bouma \& D. G. Bowhuis (Eds.), Attention and Performance X (pp. 531-556). Hillsdale, NJ: Lawrence Erlbaum Associates.

Posner, M. I., \& Peterson, S. E. (1990). The attention system of the human brain. Annual Review of Neuroscience, 13, 25-42. 
Posner, M. I., Rafal, R. D., Choate, L., \& Vaughan, J. (1985). Inhibition of return: Neural basis and function. Cognitive Neuropsychology, 2, 211-228.

Posner, M. I., Snyder, C. R. R., \& Davidson, B. J. (1980). Attention and the detection of signals. Journal of Experimental Psychology: General, 109, 160-174.

Pratt, J., Kingstone, A., \& Khoe, W. (1997). Inhibition of return in location- and identity-based choice decision tasks. Perception \& Psychophysics, 59, 964-971.

Rafal, R. (1996). Visual attention: Converging operations from neurology and psychology. In A. F. Kramer, M. G. H. Coles \& G. D. Logan (Eds.), Converging operations in the study of visual selective attention (pp. 139-192). Washington, DC: American Psychological Association.

Rafal, R. D., Calabresi, P. A., Brennan, C. W., \& Sciolto, T. K. (1989). Saccade preparation inhibits reorienting to recently attended locations. Journal of Experimental Psychology: Human Perception and Performance, 15, 673-685.

Scaife, M., \& Bruner, J. S. (1975). The capacity for joint visual attention in the infant. Nature, 253, 265-266.

Tantam, D. (1992). Characterizing the fundamental social handicap in autism. Acta Paedopsychiatrica, 55, 88-91.

Valentine, T., \& Bruce, V. (1986). The effect of race, inversion and encoding activity upon face recognition. Acta Psychologica, 61, 259-273.

Vecera, S. P., \& Johnson, M. H. (1995). Gaze detection and the cortical processing of faces: Evidence from infants and adults. Visual Cognition, 2, 59-87.

Walker-Smith, G. J., Gale, A. G., \& Findlay, J. M. (1977). Eye movement strategies involved in face perception. Perception, 6, 313-326. 
Walsh, V., \& Perrett, D. I. (1994). Visual attention in the occipitotemporal processing stream of the macaque. Cognitive Neuropsychology, 11, 243-263.

Yantis, S., \& Jonides, J. (1984). Abrupt visual onsets and selective attention: Evidence from visual search. Journal of Experimental Psychology: Human Perception and Performance, 10, 601-621.

Yarbus, A. L. (1967). Eye movements and vision. New York: Plenum Press.

Yin, R. K. (1969). Looking at upside down faces. Journal of Experimental Psychology, 81, 141. 


\section{Author Notes}

The authors thank Jon Driver for helpful discussions of his work on this topic, as well as Ray Klein, Luis Fuentes and two anonymous reviewers for comments on an earlier version of the manuscript. Correspondence concerning this article should be addressed to Stephen R. H. Langton, Department of Psychology, University of Stirling, Stirling, FK9 4LA, Scotland. Electronic mail may be sent via the Internet to srhl1@stirling.ac.uk. 